\title{
The prognostic value of ERCCI and RRMI gene expression in completely resected non-small cell lung cancer: tumor recurrence and overall survival
}

This article was published in the following Dove Press journal:

Cancer Management and Research

2 October 2013

Number of times this article has been viewed

\section{Apichat Tantraworasin' \\ Somcharoen Saeteng' \\ Nirush Lertprasertsuke ${ }^{2}$ \\ Nuttapon Arayawudhikul ${ }^{3}$ \\ Choosak Kasemsarn ${ }^{4}$ \\ Jayanton Patumanond ${ }^{5}$}

'General Thoracic Unit, Department of Surgery, Faculty of Medicine, Chiang Mai University Hospital, Chiang Mai, Thailand; ${ }^{2}$ Department of Pathology, Faculty of Medicine, Chiang Mai University Hospital, Chiang Mai, Thailand; ${ }^{3}$ Cardiovascular Thoracic Unit, Department of Surgery, Lampang Hospital, Lampang, Thailand; ${ }^{4} \mathrm{Cardiovascular}$ Thoracic Unit, Department of Surgery, Chest Institute, Nonthaburi, Thailand; ${ }^{5}$ Department of Community Medicine, Faculty of Medicine, Chiang Mai University Hospital, Chiang Mai, Thailand
Correspondence: Somcharoen Saeteng General Thoracic Surgery Unit, Department of Surgery, Faculty of Medicine, 110 Intawaroroj Road, Chiang Mai University Hospital, Chiang Mai, Thailand 50200

Tel +66 8I5954549

Fax +6653946139

Email tengearneae@gmail.com
Background: The roles of excision repair cross-complementing group 1 gene (ERCC1) expression and ribonucleotide reductase subunit M1 gene (RRM1) expression in completely resected non-small cell lung cancer (NSCLC) are still debatable. Previous studies have shown that both genes affected the overall survival and outcomes of patients who received platinumbased chemotherapy; however, some studies did not show this correlation. The aim of this study was to evaluate the prognostic values of $E R C C 1$ and $R R M 1$ gene expression in predicting tumor recurrence and overall survival in patients with completely resected NSCLC who received adjuvant chemotherapy and in those who did not.

Patients and methods: A retrospective cohort study was conducted in 247 patients with completely resected NSCLC. All patients had been treated with anatomic resection (lobectomy or pneumonectomy) with systematic mediastinal lymphadenectomy between January 2002 and December 2011 at Chiang Mai University Hospital, Chiang Mai, Thailand. They were divided into two groups: recurrence and no recurrence. Protein expression of ERCC1 and RRM1 was determined by immunohistochemistry. Correlations between clinicopathologic variables, including ERCC1 and RRM1 expression and tumor recurrence, were analyzed. Univariate and multivariate Cox proportional hazards regression analysis stratified by nodal involvement, tumor staging, intratumoral blood vessel invasion, intratumoral lymphatic invasion, and tumor necrosis was used to identify the prognostic roles of ERCC1 and RRM1.

Results: ERCC1 and RRM1 expression did not demonstrate prognostic value for tumor recurrence and overall survival in patients with completely resected NSCLC. In patients who did not receive adjuvant chemotherapy treatment, those with high ERCC1 and high RRM1 expression seemed to have greater potential for tumor recurrence and shorter overall survival than did those who had low ERCC1 and low RRM1 (hazard ratio [HR] $=1.7,95 \%$ confidence interval $[\mathrm{CI}]=0.6-4.3, P=0.292$ and $\mathrm{HR}=1.6,95 \% \mathrm{CI}=0.5-4.5, P=0.411$, respectively). In contrast, in patients who received adjuvant chemotherapy treatment, those with high ERCC1 and high RRM1 expression seemed to have benefited from adjuvant chemotherapy and showed good overall survival compared with those who had low ERCC1 and low RRM1 (HR $=0.8,95 \%$ $\mathrm{CI}=0.4-1.8, P=0.612$ and $\mathrm{HR}=0.4,95 \% \mathrm{CI}=0.1-2.4, P=0.325$, respectively). Subgroup analysis in patients whose first-line metastatic chemotherapy failed demonstrated that ERCC1 expression and RRM1 expression were not prognostic factors for tumor recurrence and overall survival; however, patients who had high ERCC1 and high RRM1 expression seemed to have benefited from first-line chemotherapy treatment ( $\mathrm{HR}=0.7,95 \% \mathrm{CI}=0.3-1.8, P=0.458$ ).

Conclusion: ERCC1 expression and RRM1 expression were not prognostic of tumor recurrence and overall survival in patients with completely resected NSCLC, either with or without adjuvant chemotherapy. Prospective studies that include a larger number of patients are needed for definite conclusions.

Keywords: ERCC1, RRM1, tumor recurrence, prognostic factor, NSCLC 


\section{Introduction}

Excision repair cross-complementing group 1 gene (ERCC1) is located on chromosome 19q13.2-q13.3 and contains 10 exons. This gene has many functions. First, it recognizes and incises branched double-single DNA structures and cuts the DNA strand carrying bulky lesions, platinum adducts, or ultraviolet-induced thymine dimers. ${ }^{1}$ Second, it allows the repair of stalled DNA replication forks during the synthesis phase (S phase) of cell cycle. Third, it is linked to singlestrand break repair. ${ }^{2}$ Finally, it plays an important role in double-strand break repair, called single-strand annealing and microhomology-mediated end joining. ${ }^{3}$ In the past 15 years, ERCC1 has become recognized for a predictive or prognostic role in advanced non-small cell lung cancer (NSCLC), ${ }^{4-6}$ however, no conclusions have been reached about the relationship between ERCC1 expression and clinicopathologic variables, and ERCC1 has not been routinely tested in clinical practice in patients with completely resected NSCLC.

Ribonucleotide reductase M1 gene (RRM1), located on chromosome 11p15.4, contains 10 axons that code for 792 amino acid proteins ${ }^{7}$ and is a large catalytic subunit of ribonucleotide reductase, which is the main enzyme for de novo synthesis of most deoxyribonucleotides. ${ }^{1}$ The vital functions of this gene are responsible for de novo DNA synthesis during the $\mathrm{S}$ phase of cell cycle (DNA replication) and DNA repair processes. The prognostic value of RRM1 has been mainly focused on advanced NSCLC treated with a gemcitabinebased regimen combined with platinum compounds. ${ }^{8-10}$

The roles of ERCC1 and RRM1 in completely resected NSCLC are still debatable. The aim of this study was to identify the correlations between ERCC1 and RRM1 expression and tumor recurrence and overall survival in patients with completely resected NSCLC with or without adjuvant chemotherapy, and to study the relationship between RRM1 and these outcomes in patients who were treated with a gemcitabine-based regimen.

\section{Patients and methods}

A retrospective cohort study was conducted in consecutive patients diagnosed with NSCLC and treated with anatomic resection and systematic mediastinal lymphadenectomy (labeled according to the IASLC Staging Manual in Thoracic Oncology) ${ }^{11}$ between January 2002 and December 2011 at Chiang Mai University Hospital, Chiang Mai, Thailand. This study was reviewed and approved by the Research Ethics Committee, Faculty of Medicine, Chiang Mai University, Chiang Mai, Thailand.
Patient characteristics, including signs and symptoms, tumor pathologic reports, and follow-up status, were reviewed from the medical recording system. Patients who had a single brain metastasis and underwent a craniectomy to remove their tumor before pulmonary resection (five patients) or had an evidence of residual tumor at the resection margin (five patients) or died within the first 30 days of the surgery (three patients) were excluded from this study.

All specimens were retrieved from formalin-fixed, paraffin-embedded tissue blocks and sliced at $10 \mathrm{~mm}$ intervals. The representative areas were marked and tissue microarrays were performed. Histopathologic examination was reevaluated by a single expert pathologist. Pathologic staging was determined according to the International Association for the Study of Lung Cancer TNM staging classification of NSCLC. ${ }^{12}$ Histologic subtypes of lung cancer were determined according to World Health Organization classification $^{13}$ and the International Association for the Study of Lung Cancer/American Thoracic Society/European Respiratory Society International Multidisciplinary Classification of Lung Adenocarcinoma. ${ }^{14}$ Presence of visceral pleural invasion, intratumoral blood vessel invasion (IVI), intratumoral lymphatic invasion, tumor necrosis, and perineural invasion were defined per a previous study. ${ }^{15}$

Regarding ERCC1 and RRM1 analysis, tissue microarrays were prepared from representative areas of the cancer from formalin-fixed, paraffin-embedded blocks. Sections of 4 micrometers thickness were cut from microarray blocks and mounted on adhesive-coated or charged glass slides. An indirect immunohistochemical (IHC) method was performed with an automated Benchmark XT detection system (Ventana Medical Systems, Tuscon, AZ, USA). For ERCC1 detection, the primary antibody was mouse monoclonal antibody clone SF1 (1:200 dilution) (Thermo Fisher Scientific, Waltham, MA, USA). For RRM1 detection, the primary antibody was rabbit polyclonal antibody (1:300 dilution) (Proteintech Group Inc, Chicago, IL, USA).

ERCC1 and RRM1 expression were classified in four levels ${ }^{16}$ ( 0 referred to no expression, $1+$ referred to $1 \%-10 \%$ expression, $2+$ referred to $11 \%-50 \%$ expression, and $3+$ referred to more than $50 \%$ expression, as shown in Figures 1 and 2) and further divided in two groups, high expression $(2+$ and $3+)$ and low expression ( 0 and $1+)$. In this study, we did not perform ERCC1 mRNA expression analysis.

All patients were actively followed postoperatively at 2 weeks, at 3-6 month intervals for the first 2 years, and yearly thereafter with a computed tomography scan of the chest and upper abdomen. Patients who had pathologic nodal 

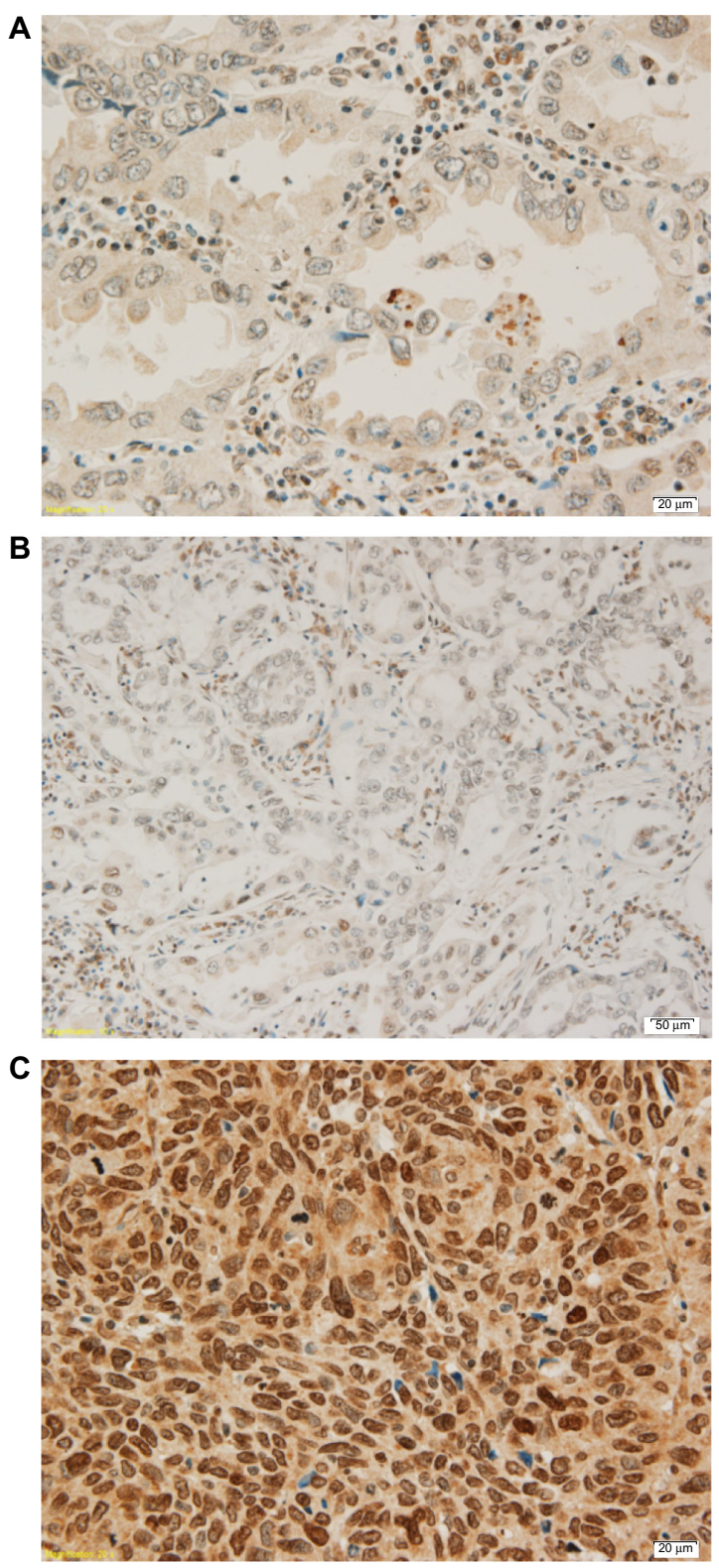

Figure I Three levels of ERCCI gene expression.

Notes: (A) ERCCI expression of adenocarcinoma I+. (B) ERCCI expression of adenocarcinoma 2+. (C) ERCCI expression of squamous cell carcinoma $3+$. Abbreviation: $\mathrm{ERCCl}$, excision repair cross-complementing group I gene.

involvement (stage IIA, IIB, IIIA and IIIB) received adjuvant platinum-based chemotherapy. If patients developed signs or symptoms that correlated with tumor recurrence or metastasis, they would be evaluated according to their signs or symptoms (ie, computed tomography brain or bone scan) and treated with first-line, second-line, and third-line of platinumbased chemotherapy according to usual clinical practice. Tumor recurrence was defined as evidence of a new lesion in the remaining lung, the hilum, the mediastinal lymph nodes, or elsewhere outside the hemithorax. The interval to recurrence was defined as the interval between the time of
A

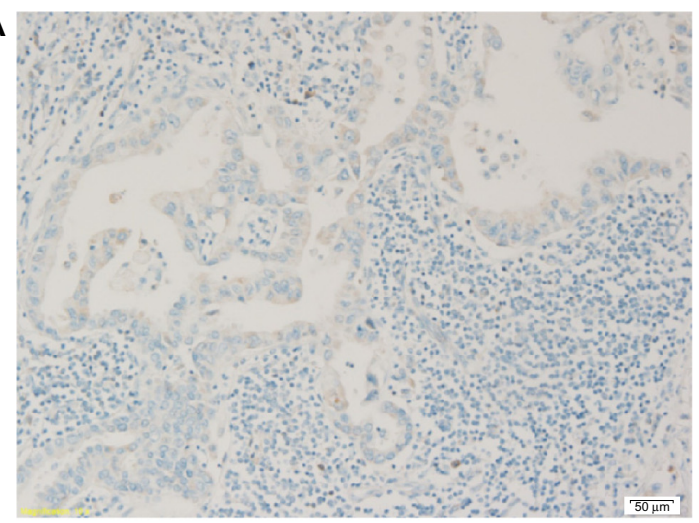

B

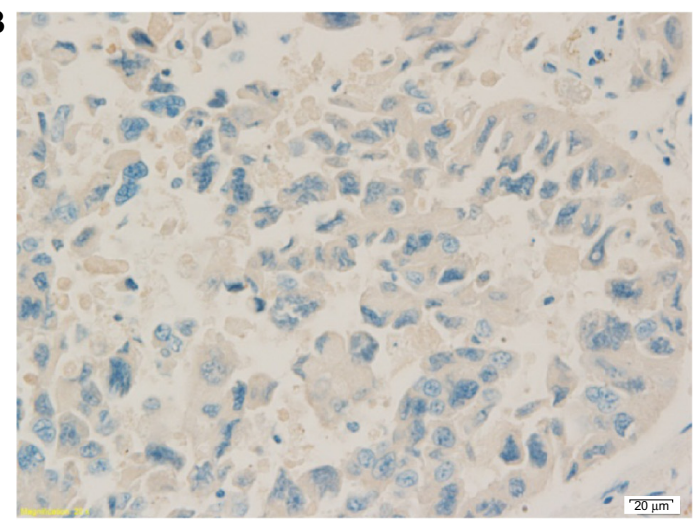

C

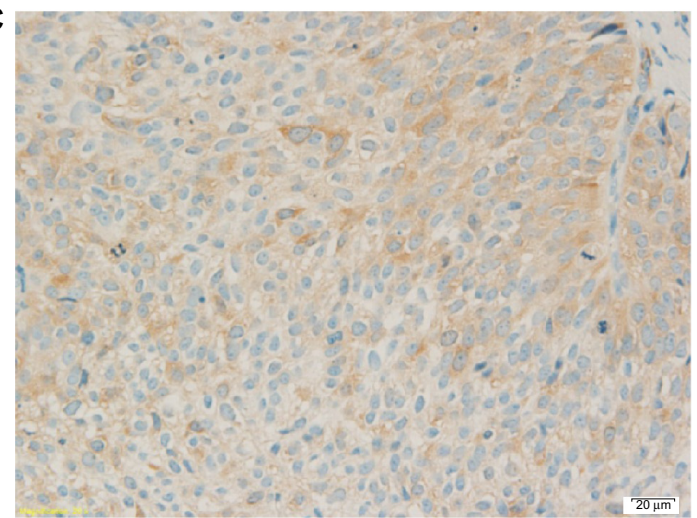

Figure 2 Three levels of RRMI gene expression.

Notes: (A) RRMI expression of adenocarcinoma I+. (B) RRMI expression of adenocarcinoma 2+. (C) RRMI expression of squamous cell carcinoma 3+. Abbreviation: RRMI, ribonucleotide reductase MI gene.

the operation and the discovery of the recurrence by means of either imaging or cytopathologic examination.

Patients were divided into two groups: recurrence and no recurrence. Categorical variables were expressed as count and percentage and analyzed by Fisher's exact test. Continuous variables were expressed as mean and standard deviation and analyzed by Student's $t$-test or Wilcoxon rank sum test. Tumor recurrence was expressed by using time zero as the date of surgery and recurrence as the end point. A Cox proportional hazards model adjusted for nodal involvement, pathologic staging, IVI, intratumoral lymphatic invasion, and 
tumor necrosis was used to identify prognostic factors of tumor recurrence, including ERCC1 and RRM1. Subgroup analysis in patients whose chemotherapy failed (including first-line, second-line, or third-line) was used to identify the prognostic values of ERCC1 and RRM1 for predicting chemotherapy failure or progression of disease after a complete course of chemotherapy. All tests were two-tailed and performed with commercial statistical software, STATA version 11.0 (StataCorp LP, College Station, TX, USA).

\section{Results}

Two hundred and forty-seven consecutive patients were enrolled in this study. The numbers of patients in the recurrence and nonrecurrence groups were 128 and 119 , respectively. The most common cell type of tumor in both groups was adenocarcinoma $(64.8 \%$ and $58 \%$, respectively). The general characteristics of patients, including age, sex, smoking status, family history of malignancy, underlying disease, and symptoms, and of surgical procedures are shown by patient group in Tables 1 and 2,

Table I General characteristics of patients with and without tumor recurrence

\begin{tabular}{|c|c|c|c|}
\hline Characteristics & $\begin{array}{l}\text { Recurrence } \\
(n=128)\end{array}$ & $\begin{array}{l}\text { Nonrecurrence } \\
(n=119)\end{array}$ & $P$-value \\
\hline Age (years) & $62.1 \pm 10.4$ & $62.9 \pm 10.1$ & 0.513 \\
\hline Sex & & & 0.513 \\
\hline Male & $75(58.6)$ & $69(58.0)$ & \\
\hline Female & $53(41.4)$ & $50(42.0)$ & \\
\hline Smoking & & & 0.259 \\
\hline Never smoked & $27(2 \mid . I)$ & $31(26.1)$ & \\
\hline Stopped smoking & $90(70.3)$ & $82(68.9)$ & \\
\hline Active smokers & $7(5.5)$ & $6(5.0)$ & \\
\hline Passive smokers & $4(3.1)$ & $0(0.0)$ & \\
\hline Packs per year & $19.7 \pm 16.1$ & $20.7 \pm 17.8$ & 0.646 \\
\hline $\begin{array}{l}\text { Family history } \\
\text { of malignancy }\end{array}$ & $7(5.5)$ & $10(8.4)$ & 0.255 \\
\hline \multicolumn{4}{|l|}{ Underlying diseases } \\
\hline Chronic lung disease & $21(16.4)$ & $21(17.7)$ & 0.464 \\
\hline Diabetic mellitus & $14(10.9)$ & $16(13.5)$ & $0.34 I$ \\
\hline $\begin{array}{l}\text { Essential } \\
\text { hypertension }\end{array}$ & $51(39.8)$ & $40(33.6)$ & 0.189 \\
\hline Dyslipidemia & $19(14.8)$ & $18(15.1)$ & 0.546 \\
\hline \multicolumn{4}{|l|}{ Symptoms } \\
\hline Hemoptysis & $45(35.2)$ & $45(37.8)$ & $0.38 I$ \\
\hline Chronic cough & $57(44.5)$ & $48(40.3)$ & 0.296 \\
\hline Poor appetite & $12(9.4)$ & $15(12.6)$ & 0.271 \\
\hline Significant weight & $27(21.1)$ & $35(29.4)$ & 0.087 \\
\hline \multicolumn{4}{|l|}{ loss } \\
\hline Chest pain & $10(7.8)$ & $10(8.4)$ & 0.524 \\
\hline Dyspnea & $24(18.8)$ & $18(15.1)$ & 0.279 \\
\hline Asymptomatic & $48(37.5)$ & $47(39.5)$ & 0.424 \\
\hline
\end{tabular}

Note: Values are $\mathrm{n}(\%)$ or mean \pm standard deviation. Abbreviation: $\mathrm{n}$, number. respectively; no statistically significant differences were found between groups. The numbers of patients who had a pathologic diagnosis of stage IIIA cancer, postive nodal involvement, or IVI were significantly higher in the recurrence group than in the nonrecurrence group $(P=0.004$, $P<0.001$, and $P=0.004$, respectively), as shown in Table 3 . The lung was the most common tumor recurrence site (52.3\%); the second was brain; and the third was bone, as shown in Table 4.

The univariate analysis of parameters for tumor recurrence demonstrated that stage IIIA cancer (hazard ratio $[\mathrm{HR}]=3.4$, $95 \%$ confidence interval $[\mathrm{CI}]=1.8-6.4, P<0.001)$, positive nodal presence $(\mathrm{HR}=2.0,95 \% \mathrm{CI}=1.4-2.8, P<0.001)$, and presence of IVI $(\mathrm{HR}=1.8,95 \% \mathrm{CI}=1.3-2.5, P=0.001)$ were significant prognostic factors of tumor recurrence (Table 5), whereas high ERCC1 expression and high RRM1 expression were not prognostic factors of tumor recurrence. As shown in Table 6, multivariate analysis indicated that ERCC1 and RRM1 were not prognostic factors of tumor recurrence, either in patients who were treated with adjuvant chemotherapy or in those who were not. Moreover, ERCC1 and RRM1 expression did not affect overall survival in patients

Table 2 Surgical procedure and chemotherapy of patients with and without tumor recurrence

\begin{tabular}{|c|c|c|c|}
\hline $\begin{array}{l}\text { Procedure and } \\
\text { chemotherapy }\end{array}$ & $\begin{array}{l}\text { Recurrence } \\
(n=\mid 28)\end{array}$ & $\begin{array}{l}\text { Nonrecurrence } \\
(n=119)\end{array}$ & $P$-value \\
\hline \multicolumn{4}{|l|}{ Surgical procedures } \\
\hline Lobectomy & II 5 (89.8) & I0I (84.9) & 0.403 \\
\hline $\begin{array}{l}\text { Bilobectomy } \\
\text { (RUL and RML) }\end{array}$ & $4(3.1)$ & $3(2.5)$ & \\
\hline $\begin{array}{l}\text { Bilobectomy } \\
\text { (RLL and RML) }\end{array}$ & $8(6.3)$ & $\mathrm{II}(9.2)$ & \\
\hline Pneumonectomy & $\mathrm{I}(0.8)$ & $4(3.4)$ & \\
\hline \multicolumn{4}{|l|}{ Chemotherapy } \\
\hline No chemotherapy & $60(46.9)$ & 7I (59.7) & 0.129 \\
\hline $\begin{array}{l}\text { Adjuvant } \\
\text { chemotherapy }\end{array}$ & $60(46.9)$ & $43(36.1)$ & \\
\hline $\begin{array}{l}\text { Neoadjuvant } \\
\text { chemotherapy }\end{array}$ & $8(6.3)$ & $5(4.2)$ & \\
\hline \multicolumn{4}{|c|}{ Chemotherapy regimens } \\
\hline $\begin{array}{l}\text { Paclitaxel plus } \\
\text { platinum }\end{array}$ & $31(47.0)$ & $27(56.3)$ & 0.914 \\
\hline $\begin{array}{l}\text { Docetaxel plus } \\
\text { platinum }\end{array}$ & $10(15.2)$ & $5(10.4)$ & \\
\hline $\begin{array}{l}\text { Navelbine plus } \\
\text { platinum }\end{array}$ & II (16.7) & $7(14.6)$ & \\
\hline $\begin{array}{l}\text { Gemcitabine plus } \\
\text { platinum }\end{array}$ & $3(4.6)$ & $2(4.2)$ & \\
\hline $\begin{array}{l}\text { Other platinum- } \\
\text { based regimens }\end{array}$ & II (16.7) & $7(14.6)$ & \\
\hline
\end{tabular}

Note: Values are $\mathrm{n}(\%)$.

Abbreviatons: $n$, number; RUL, right upper lobe; RML, right middle lobe. 
Table 3 Histopathologic reports of patients with and without tumor recurrence

\begin{tabular}{|c|c|c|c|}
\hline Reports & $\begin{array}{l}\text { Recurrence } \\
(n=128)\end{array}$ & $\begin{array}{l}\text { Nonrecurrence } \\
(n=I \mid 9)\end{array}$ & $P$-value \\
\hline Histologic types & & & 0.465 \\
\hline Adenocarcinoma & $83(64.8)$ & $69(58.0)$ & \\
\hline $\begin{array}{l}\text { Squamous cell } \\
\text { carcinoma }\end{array}$ & $31(24.2)$ & $37(3 \mathrm{I} .1)$ & \\
\hline Others* & $14(10.9)$ & $13(10.9)$ & \\
\hline Tumor grading & & & 0.544 \\
\hline Well differentiated & $48(37.5)$ & $34(28.6)$ & \\
\hline $\begin{array}{l}\text { Moderately } \\
\text { differentiated }\end{array}$ & $52(40.6)$ & $55(46.2)$ & \\
\hline Poorly differentiated & $20(15.6)$ & $19(16.0)$ & \\
\hline Undifferentiated & $3(2.3)$ & $7(5.9)$ & \\
\hline $\begin{array}{l}\text { Mucinous type of } \\
\text { adenocarcinoma in situ }\end{array}$ & $3(2.3)$ & $2(1.7)$ & \\
\hline $\begin{array}{l}\text { Nonmucinous type of } \\
\text { adenocarcinoma in situ }\end{array}$ & $2(1.6)$ & $2(1.7)$ & \\
\hline Pathologic staging & & & 0.004 \\
\hline IA & $12(9.4)$ & $25(21.0)$ & \\
\hline IB & $22(17.2)$ & $25(2 I .0)$ & \\
\hline IIA & $24(18.8)$ & $21(17.7)$ & \\
\hline IIB & $15(11.7)$ & $21(17.7)$ & \\
\hline IIIA & $53(41.4)$ & $24(20.2)$ & \\
\hline IIIB & $2(1.6)$ & $3(2.5)$ & \\
\hline Tumor diameter & & & 0.340 \\
\hline$\leq 5 \mathrm{~cm}$ & $84(65.6)$ & $82(68.9)$ & \\
\hline$>5 \mathrm{~cm}$ & $44(34.4)$ & $37(31.1)$ & \\
\hline Nodal involvement & & & $<0.001$ \\
\hline Nodal negative & 61 (47.7) & $83(69.8)$ & \\
\hline Nodal positive ${ }^{\dagger}$ & $67(52.3)$ & $36(30.3)$ & \\
\hline Tumor necrosis & $55(43.0)$ & $46(38.7)$ & 0.288 \\
\hline Visceral pleural invasion & $27(2 I . I)$ & $21(17.7)$ & 0.301 \\
\hline Neural invasion & $7(5.5)$ & $3(2.5)$ & 0.198 \\
\hline $\begin{array}{l}\text { Intratumoral lymphatic } \\
\text { invasion }\end{array}$ & II 2 (87.5) & $95(79.8)$ & 0.072 \\
\hline $\begin{array}{l}\text { Intratumoral blood } \\
\text { vessel invasion }\end{array}$ & $63(49.2)$ & $38(31.9)$ & 0.004 \\
\hline ERCCI expression & & & 0.272 \\
\hline Low expression & $77(60.2)$ & 77 (64.7) & \\
\hline High expression & $51(39.8)$ & $42(35.3)$ & \\
\hline RRMI expression & & & 0.375 \\
\hline Low expression & $81(63.3)$ & $72(60.5)$ & \\
\hline High expression & $47(36.7)$ & $47(39.5)$ & \\
\hline
\end{tabular}

Notes: Values are $\mathrm{n}(\%)$. Other cell types included adenocarcinoma in situ, large cell carcinoma, adenoid cystic carcinoma,lymphoepithelioma-like carcinoma, and adenosquamous cell carcinoma; 'nodal positive refers to the presenting of malignant cells in any node level $(1-14)$.

Abbreviations: $\mathrm{n}$, number; $\mathrm{ERCCI}$, excision repair cross-complementing group I gene; RRMI, ribonucleotide reductase MI gene.

with completely resected NSCLC with or without adjuvant chemotherapy, as shown in Table 7. There were no correlations between RRM1 expression and outcomes in patients who were treated with a gemcitabine-based regimen in terms of disease progression after receiving first-line treatment (HR $=1.0,95 \% \mathrm{CI}=0.6-1.7, P=1.000)$.
Table 4 Sites of tumor recurrence (metastases)

\begin{tabular}{lll}
\hline Sites & Number of patients & Percent \\
\hline Lung & 67 & 52.3 \\
Brain & 38 & 29.7 \\
Bone & 22 & 17.2 \\
Mediastinal lymph node & 10 & 7.8 \\
Pleura & 8 & 6.3 \\
Supraclavicular lymph node & 7 & 5.5 \\
Adrenal gland & 6 & 4.7 \\
Liver & 4 & 3.1 \\
Skin & 4 & 3.1 \\
Chest wall & 3 & 2.3 \\
Spleen & 2 & 1.6 \\
Kidney & 1 & 0.8 \\
Cervical lymph node & 1 & 0.8 \\
Stomach & 1 & 0.8 \\
Groin node & 1 & 0.8
\end{tabular}

Subgroup analysis of the prognostic value of ERCC1 and RRM1 expression to predict chemotherapy failure or progression of disease after a complete course of chemotherapy demonstrated that ERCC1 expression and RRM1 expression were not prognostic factors of failed chemotherapy in univariate and multivariate models, as shown in Tables 8 and 9 , respectively.

\section{Discussion}

$E R C C 1$ is a DNA repair gene coding $5^{\prime}$ endonuclease in nucleotide excision repair pathway and has a vital rule in genomic stability, which is an important step in lung cancer pathogenesis. ${ }^{17}$ For 15 years, ERCC1 has been recognized for its prognostic role in advanced NSCLC; ${ }^{46}$ however, no conclusions have been reached regarding the relationship between ERCC1 expression and clinical outcomes (tumor recurrence and overall survival) in patients with completely resected NSCLC. In our study, univariate and multivariate analyses revealed no statistically significant differences between ERCC1 expression and tumor recurrence or overall survival in patients with completely resected NSCLC who received or did not receive adjuvant chemotherapy treatment. However, patients who had not received adjuvant chemotherapy and who had low ERCC1 expression tended to survive longer than those with high ERCC1 expression (HR $=0.8,95 \%$ $\mathrm{CI}=0.3-2.0, P=0.596$ ), whereas patients who had received adjuvant chemotherapy and who had high ERCC1 expression tended to survive longer than those with low ERCC1 expression $(\mathrm{HR}=0.4,95 \% \mathrm{CI}=0.1-2.4, P=0.325)$. The trends in our results corresponded with several previous studies. Pesta et a $1^{18}$ reported a group of 90 patients with NSCLC who underwent curative lung resection and concluded that patients who 
Table 5 Univariate hazard ratios and $95 \%$ confidence intervals of tumor recurrence for parameters with clinical and statistical significance

\begin{tabular}{|c|c|c|c|}
\hline Parameters & HR & $95 \% \mathrm{Cl}$ & $P$-value \\
\hline Age $>70$ years & 1.0 & $0.7-1.5$ & 0.965 \\
\hline Male & I.I & $0.8-1.5$ & 0.654 \\
\hline \multicolumn{4}{|l|}{ Smoking } \\
\hline Never smoked & Reference & & \\
\hline Stopped smoking & 1.4 & $0.9-2.2$ & 0.107 \\
\hline Active smokers & 1.4 & $0.6-3.1$ & 0.470 \\
\hline Passive smokers & 1.8 & $0.6-5.2$ & 0.259 \\
\hline \multicolumn{4}{|l|}{ Histologic types } \\
\hline Others* & Reference & & \\
\hline Adenocarcinoma & 1.0 & $0.6-1.8$ & 0.962 \\
\hline Squamous cell carcinoma & 0.8 & $0.4-1.5$ & 0.507 \\
\hline \multicolumn{4}{|l|}{ Tumor grading } \\
\hline Well differentiated & Reference & & \\
\hline Moderately differentiated & 0.9 & $0.6-1.3$ & 0.432 \\
\hline Poorly differentiated & 0.9 & $0.5-1.5$ & 0.671 \\
\hline Undifferentiated & 0.4 & $0.1-1.4$ & 0.160 \\
\hline $\begin{array}{l}\text { Mucinous type of } \\
\text { adenocarcinoma in situ }\end{array}$ & 0.9 & $0.3-2.7$ & 0.785 \\
\hline $\begin{array}{l}\text { Nonmucinous type of } \\
\text { adenocarcinoma in situ }\end{array}$ & 0.9 & $0.2-3.9$ & $0.94 I$ \\
\hline \multicolumn{4}{|l|}{ Pathologic staging } \\
\hline IA & Reference & & \\
\hline IB & 1.7 & $0.8-3.5$ & 0.136 \\
\hline IIA & 2.1 & $1.1-4.2$ & 0.039 \\
\hline IIB & 1.9 & $0.9-4.0$ & 0.102 \\
\hline IIIA & 3.4 & $1.8-6.4$ & $<0.001$ \\
\hline IIIB & 2.3 & $0.5-10.1$ & 0.284 \\
\hline Tumor diameter $>5 \mathrm{~cm}$ & 1.3 & $0.9-1.9$ & 0.146 \\
\hline Nodal positive ${ }^{\dagger}$ & 2.0 & $1.4-2.8$ & $<0.001$ \\
\hline Tumor necrosis & 1.3 & $0.9-1.9$ & 0.103 \\
\hline Visceral pleural invasion & 1.2 & $0.8-1.9$ & 0.376 \\
\hline Neural invasion & 1.6 & $0.8-3.5$ & 0.214 \\
\hline Intratumoral lymphatic invasion & 1.6 & $0.9-2.6$ & 0.099 \\
\hline Intratumoral blood vessel invasion & 1.8 & $1.3-2.5$ & 0.001 \\
\hline High ERCCI expression & 1.2 & $0.8-1.7$ & 0.295 \\
\hline High RRMI expression & 0.9 & $0.6-1.3$ & 0.673 \\
\hline \multicolumn{4}{|l|}{ Chemotherapy } \\
\hline No chemotherapy & Reference & & \\
\hline Adjuvant chemotherapy & 1.4 & $0.9-1.9$ & 0.086 \\
\hline Neoadjuvant chemotherapy & 1.4 & $0.7-3.0$ & 0.344 \\
\hline
\end{tabular}

Notes: *Other cell types included adenocarcinoma in situ, large cell carcinoma, adenoid cystic carcinoma, lymphoepithelioma-like carcinoma, and adenosquamous cell carcinoma; 'nodal positive refers to the presenting of malignant cells in any node level (I-I4).

Abbreviations: $\mathrm{HR}$, hazard ratio; $\mathrm{Cl}$, confidence interval; $\mathrm{ERCCI}$, excision repair cross-complementing group I gene; RRMI, ribonucleotide reductase MI gene.

had been treated with adjuvant chemotherapy and had shown low expression of ERCC1 had adverse prognoses. Many previous studies reported converse results. In 2008, a large retrospective cohort study of the International Adjuvant Lung Trial-bio study enrolled 867 patients with resected NSCLC; ${ }^{19}$ using IHC, the researchers found that ERCC1-negative tumor produced a significantly prolonged survival in patients who
Table 6 Multivariate hazard ratios and 95\% confidence intervals of tumor recurrence for ERCCI and RRMI expression

\begin{tabular}{|c|c|c|c|c|}
\hline $\begin{array}{l}\text { ERCCI } \\
\text { expression }\end{array}$ & $\begin{array}{l}\text { RRMI } \\
\text { expression }\end{array}$ & HR & $95 \% \mathrm{Cl}$ & $P$-value \\
\hline \multicolumn{5}{|c|}{ Without adjuvant chemotherapy } \\
\hline Low & Low & Reference & & \\
\hline Low & High & 0.6 & $0.3-1.6$ & 0.360 \\
\hline High & Low & 1.4 & $0.5-3.5$ & 0.496 \\
\hline High & High & 1.7 & $0.6-4.3$ & 0.292 \\
\hline \multicolumn{5}{|c|}{ With adjuvant chemotherapy } \\
\hline Low & Low & Reference & & \\
\hline Low & High & I.I & $0.5-2.6$ & 0.818 \\
\hline High & Low & 1.4 & $0.6-3.2$ & 0.471 \\
\hline High & High & 0.8 & $0.4-1.8$ & 0.612 \\
\hline
\end{tabular}

Note: All analyses adjusted for nodal involvement, pathologic staging, intratumoral blood vessel invasion, intratumoral lymphatic invasion, and tumor necrosis.

Abbreviations: $\mathrm{ERCCl}$, excision repair cross-complementing group I gene; RRMI, ribonucleotide reductase $\mathrm{MI}$ gene; $\mathrm{HR}$, hazard ratio; $\mathrm{Cl}$, confidence interval.

had received adjuvant chemotherapy (adjusted $\mathrm{HR}=0.65$, $95 \% \mathrm{CI}=0.50-0.86, P=0.002$ ), whereas patients who did not received adjuvant chemotherapy and had ERCC1-positive tumors survived longer than those with ERCC1-negative tumors (adjusted HR $=0.66,95 \% \mathrm{CI}=0.49-0.90, P=0.009$ ). They concluded that patients with completely resected NSCLC and ERCC1-negative tumors appeared to benefit from adjuvant cisplatin-based chemotherapy, whereas patients with ERCC1-positive tumors did not. Lee et $\mathrm{al}^{20}$ demonstrated that patients with a positive ERCC1 expression without adjuvant chemotherapy survived longer than ERCC1-negative patients (median overall survival 7.6 years for ERCC1positive versus 4.0 years for ERCC1-negative, $P=0.046$ ) and concluded that ERCC1 expression was a positive prognostic marker in resected NSCLC. Bepler et $\mathrm{al}^{21}$ reported that low ERCC1 indicated a significant benefit for adjuvant chemotherapy $(\mathrm{HR}=0.73$ for chemotherapy versus control, $P=0.02)$.

Table 7 Multivariate hazard ratios and 95\% confidence intervals of death for ERCCI and RRMI expression

\begin{tabular}{|c|c|c|c|c|}
\hline $\begin{array}{l}\text { ERCCI } \\
\text { expression }\end{array}$ & $\begin{array}{l}\text { RRMI } \\
\text { expression }\end{array}$ & HR & $95 \% \mathrm{Cl}$ & $P$-value \\
\hline \multicolumn{5}{|c|}{ Without adjuvant chemotherapy } \\
\hline Low & Low & Reference & & \\
\hline Low & High & 0.8 & $0.3-2.0$ & 0.596 \\
\hline High & Low & 1.6 & $0.5-4.5$ & $0.41 \mathrm{I}$ \\
\hline High & High & 1.1 & $0.4-2.8$ & 0.876 \\
\hline \multicolumn{5}{|c|}{ With adjuvant chemotherapy } \\
\hline Low & Low & Reference & & \\
\hline Low & High & 1.6 & $0.1-18.5$ & 0.696 \\
\hline High & Low & 2.3 & $0.6-9.2$ & 0.222 \\
\hline High & High & 0.4 & $0.1-2.4$ & 0.325 \\
\hline
\end{tabular}

Note: All analyses adjusted for nodal involvement, pathologic staging, intratumoral blood vessel invasion, intratumoral lymphatic invasion, and tumor necrosis.

Abbreviations: $\mathrm{ERCCl}$, excision repair cross-complementing group I gene; RRMI, ribonucleotide reductase $\mathrm{MI}$ gene; $\mathrm{HR}$, hazard ratio; $\mathrm{Cl}$, confidence interval. 
Table 8 Univariate hazard ratios and $95 \%$ confidence intervals of failed chemotherapy after tumor recurrence for parameters with clinical and statistical significance

\begin{tabular}{|c|c|c|c|}
\hline Parameters & HR & $95 \% \mathrm{Cl}$ & $P$-value \\
\hline Age $>70$ years & 0.9 & $0.4-1.8$ & 0.684 \\
\hline Male & 1.0 & $0.6-1.7$ & 0.907 \\
\hline \multicolumn{4}{|l|}{ Smoking } \\
\hline Never smoked & Reference & & \\
\hline Stopped smoking & 0.9 & $0.5-1.6$ & 0.738 \\
\hline Active smokers & 0.5 & $0.1-2.1$ & 0.354 \\
\hline Passive smokers & 0.6 & $0.1-4.3$ & 0.591 \\
\hline \multicolumn{4}{|l|}{ Histologic types } \\
\hline Others* & Reference & & \\
\hline Adenocarcinoma & 1.0 & $0.4-2.3$ & 0.980 \\
\hline Squamous cell carcinoma & 0.8 & $0.3-2.2$ & 0.686 \\
\hline \multicolumn{4}{|l|}{ Tumor grading } \\
\hline Well differentiated & Reference & & \\
\hline Moderately differentiated & I.I & $0.6-1.9$ & 0.757 \\
\hline Poorly differentiated & 0.5 & $0.1-2.0$ & 0.316 \\
\hline Undifferentiated & - & - & - \\
\hline $\begin{array}{l}\text { Mucinous type of } \\
\text { adenocarcinoma in situ }\end{array}$ & 1.8 & $0.4-8.0$ & 0.392 \\
\hline $\begin{array}{l}\text { Nonmucinous type of } \\
\text { adenocarcinoma in situ }\end{array}$ & I.I & $0.4-3.2$ & 0.874 \\
\hline \multicolumn{4}{|l|}{ Pathologic staging } \\
\hline IA & Reference & & \\
\hline IB & 0.8 & $0.3-2.2$ & 0.638 \\
\hline$\| \mathrm{A}$ & 1.4 & $0.5-3.9$ & 0.472 \\
\hline IIB & 1.7 & $0.6-4.8$ & 0.315 \\
\hline IIIA & 1.0 & $0.4-2.4$ & 0.910 \\
\hline IIIB & 1.8 & $0.4-7.3$ & 0.411 \\
\hline Tumor diameter $>5 \mathrm{~cm}$ & 1.7 & $0.9-2.9$ & 0.083 \\
\hline Nodal positive ${ }^{\dagger}$ & 1.0 & $0.6-1.6$ & 0.835 \\
\hline Tumor necrosis & 0.7 & $0.4-1.3$ & 0.307 \\
\hline Visceral pleural invasion & 1.0 & $0.5-1.9$ & 0.927 \\
\hline Neural invasion & 1.2 & $0.3-4.8$ & 0.841 \\
\hline Intratumoral lymphatic invasion & 0.8 & $0.4-1.7$ & 0.532 \\
\hline Intratumoral blood vessel invasion & 1.4 & $0.8-2.4$ & 0.208 \\
\hline High ERCCI expression & 0.8 & $0.5-1.5$ & 0.509 \\
\hline High RRMI expression & 0.8 & $0.5-1.3$ & 0.381 \\
\hline \multicolumn{4}{|l|}{ Regimens of chemotherapy } \\
\hline Platinum plus other drug & Reference & & \\
\hline Platinum plus gemcitabine & 0.8 & $0.5-1.5$ & 0.509 \\
\hline
\end{tabular}

Notes: *Other cell types include: adenocarcinoma in situ, large cell carcinoma adenoid cystic carcinoma, lymphoepithelioma-like carcinoma and adenosquamous cell carcinoma; ${ }^{\dagger}$ nodal positive refers to the presenting of malignant cells in any node level (I-I4).

Abbreviations: $\mathrm{HR}$, hazard ratio; $\mathrm{Cl}$, confidence interval; $\mathrm{ERCCl}$, excision repair cross-complementing group I gene; RRMI, ribonucleotide reductase MI gene.

Custodio et $\mathrm{al}^{22}$ and Olaussen et a ${ }^{19}$ also reported that patients with ERCC1-negative tumors derived a substantial benefit from adjuvant chemotherapy, when compared with patients with ERCC1-positive tumors.

Patients with advanced disease or tumor recurrence were treated with platinum-based chemotherapy. The results from univariate and multivariate analysis in this study demonstrated that ERCC1 expression was not a predictive
Table 9 Multivariate hazard ratios and $95 \%$ confidence intervals of failed chemotherapy after tumor recurrence for ERCCI and RRMI expression

\begin{tabular}{lllll}
\hline $\begin{array}{l}\text { ERCCI } \\
\text { expression }\end{array}$ & $\begin{array}{l}\text { RRMI } \\
\text { expression }\end{array}$ & HR & 95\% Cl & P-value \\
\hline Low & Low & Reference & & \\
Low & High & 1.2 & $0.4-3.2$ & 0.782 \\
High & Low & 1.8 & $0.5-6.7$ & 0.397 \\
High & High & 0.7 & $0.3-1.8$ & 0.458 \\
\hline
\end{tabular}

Note: All analyses adjusted for nodal involvement, pathologic staging, intratumoral blood vessel invasion, intratumoral lymphatic invasion, and tumor necrosis.

Abbreviations: ERCCl, excision repair cross-complementing group I gene; RRMI, ribonucleotide reductase $\mathrm{MI}$ gene; $\mathrm{HR}$, hazard ratio; $\mathrm{Cl}$, confidence interval.

value for failed chemotherapy; however, there was a trend toward increased risk for failed chemotherapy if patients had high ERCC1 expression but had low RRM1 expression (HR $=1.8,95 \%$ CI $=0.5-6.7, P=0.397)$. Patients who had a high expression of ERCC1 and RRM1 trended to have a good response to chemotherapy $(\mathrm{HR}=0.7,95 \% \mathrm{CI}=0.3-1.8$, $P=0.458$ ). Reynolds et $\mathrm{al}^{10}$ also reported no predictive value for ERCC1 expression when assessed by IHC. Booton et $\mathrm{al}^{23}$ demonstrated that ERCC1 expression did not favor a prognostically better outcome after platinum-based chemotherapy in advanced NSCLC; however, this study identified ERCC1 by mRNA analysis, which was different from our study. Wang et al ${ }^{4}$ retrospectively reviewed 124 patients with advanced NSCLC and reported that patients with ERCC1-negative tumors but RRM1-positive tumors had a longer median survival time than those with ERCC1-positive tumors (13.4 months versus 9.1 months, $P=0.007$ ). Roth et $\mathrm{a}^{24}$ performed a systematic review and meta-analysis of 11 studies on the prognostic role of ERCC1 in advanced NSCLC and reported that patients who had high ERCC1 expression had a significantly lower response (risk ratio $=0.8$, $95 \% \mathrm{CI}=0.66-0.98)$ and a significantly higher risk for death $(\mathrm{HR}=2.04,95 \% \mathrm{CI}=1.48-2.80)$. However, a recent study by Friboulet et $\mathrm{al}^{25}$ reported using the $8 \mathrm{~F} 1$ antibody to measure the level of ERCC1 expression by means of IHC analysis in a validation set of samples obtained from 494 patients in two independent Phase III trials (the National Cancer Institute of Canada Clinical Trials Group JBR. $10^{26}$ and the Cancer and Leukemia Group B 9633 Trial from the Lung Adjuvant Cisplatin Evaluation Biology Project). ${ }^{27}$ Both trials studied the correlation between the absence of ERCC1 expression and platinum response. They mapped the epitope recognized by 16 commercially available ERCC 1 antibodies and investigated the capacity of the different ERCC1 isoforms to repair platinum-induced DNA damage. The results of their study showed that there was low validity for detecting the predictive 
effect of immunostaining for ERCC1 protein. None of the 16 antibodies could distinguish among the four ERCC1 protein isoforms, whereas only one isoform produced a protein that had full capacities for nucleotide excision repair and cisplatin resistance. They concluded that $\mathrm{IHC}$ analysis with the use of currently available ERCC1 antibodies did not specifically detect the unique functional ERCC1 isoform; therefore, the usefulness of IHC for ERCC1 in guiding therapeutic decision making was limited.

RRM1 is a region with a frequent loss of heterozygosity in NSCLC ${ }^{28}$ and is involved in tumor invasiveness and metastasis. ${ }^{29}$ The important mediator that affects RRM1 to regulate cellular signaling, survival, and migration is phosphatase and tensin homologue. ${ }^{22}$ In our study, RRM1 expression had no prognostic value for tumor recurrence or overall survival in patients with completely resected NSCLC, whether they had received adjuvant chemotherapy or not. Moreover, there was no correlation between RRM1 expression and disease progression in patients treated with a gemcitabine-based regimen $(\mathrm{HR}=1.0,95 \% \mathrm{CI}=0.6-1.7$, $P=1.000)$. However, patients who did not receive adjuvant chemotherapy with high RRM1 and ERCC1 expression tended to have more potential for tumor recurrence than those with low RRM1 and ERCC1 expression $(\mathrm{HR}=1.7$, $95 \% \mathrm{CI}=0.6-4.3, P=0.292$ ), whereas patients who received adjuvant chemotherapy and who had high RRM1 and ERCC1 expression tended to benefit from adjuvant chemotherapy ( $\mathrm{HR}=0.8,95 \% \mathrm{CI}=0.4-1.8, P=0.612$ ). About overall survival, patients with high RRM1 and ERCC1 expression who received adjuvant chemotherapy tended to have a longer survival than those with low RRM1 and ERCC1 expression ( $\mathrm{HR}=0.4,95 \% \mathrm{CI}=0.1-2.4, P=0.325)$. Subgroup analysis of failed chemotherapy after treatment with first-line, second-line, or third-line chemotherapy demonstrated that patients with high RRM1 and ERCC11 expression tend to benefit from chemotherapy $(\mathrm{HR}=0.7,95 \% \mathrm{CI}=0.3-1.8$, $P=0.458)$. RRM1 expression did not affect outcomes of patients who were treated with a gemcitabine-based regimen; however, in this study, only 14 patients $(12 \%)$ received a gemcitabine-based regimen (ten patients for first-line and two patients for second-line chemotherapy). In previous studies, high RRM1 expression was associated with prolonged survival in NSCLC patients. ${ }^{30}$ Recently, Bepler et $\mathrm{al}^{31}$ performed randomized international Phase III trials of ERCC1 and RRM1 expression-based chemotherapy versus gemcitabine/carboplatin in advanced NSCLC and demonstrated that patients in both treatment arms with low ERCC1 and RRM1 expression who received the same treatment (the control arm was gemcitabine/carboplatin and the experimental arm was docetaxel/carboplatin) had a statistically better progression-free survival (8.1 months in the control arm and 5.0 months in experimental arm, $P=0.02$ ). Gong et $a^{32}$ reported a meta-analysis of 18 studies of the correlation between RRM1 expression and clinical outcomes of gemcitabine-containing regimen in advanced NSCLC $(n=1,243)$ and found that patients with low/negative RRM1 expression had a significantly higher response rate to a gemcitabine-containing regimen (odds ratio $=0.31,95 \% \mathrm{CI}=21-0.45, P<0.001)$. Patients with low/negative RRM1 who were treated with a gemcitabinecontaining regimen survived 3.94 months longer $(95 \%$ $\mathrm{CI}=2.15-5.73, P<0.001)$ and had a longer time of progression (2.64 months; $95 \% \mathrm{CI}=0.39-4.89, P=0.02)$ than those with high/positive RRM1. They concluded that low/negative RRM1 expression was associated with better response to a gemcitabine-containing regimen and better prognosis in patients with advanced NSCLC. A few previous studies focused on patients with early-stage NSCLC who were treated with surgery only. Zheng et $\mathrm{al}^{33}$ retrospectively reviewed 187 patients with completely resected NSCLC without adjuvant chemotherapy and reported that a low RRM1 expression was associated with a median overall survival of more than 60 months, and high RRM1 expression with more than 120 months.

Limitations of this study include the retrospective nature of the study, a small number of patients who received a gemcitabine-containing regimen, and the small sample size.

\section{Conclusion}

Our study demonstrated that ERCC and RRM1 expression have no prognostic value for tumor recurrence and overall survival, either in the setting of patients with completely resected NSCLC with or without platinum-based adjuvant chemotherapy or patients with failed first-line, second-line, or third-line chemotherapy. Furthermore, ERCC1 and RRM1 expression do not have prognostic value for the benefits of gemcitabine-containing regimen. Our results contrast with previous reports that high ERCC1 and RRM1 expression tend to be associated with long survival among patients with completely resected NSCLC and are also associated with an adverse response to chemotherapy. The relationship between ERCC1 expression, RRM1 expression, and clinical outcomes of chemotherapy treatment in completely resected NSCLC and advanced NSCLC still needs to be clarified. There are some ongoing clinical trials based on ERCC1 and/or RRM1 expression in NSCLC. 


\section{Acknowledgments}

This study was supported by a grant from the Faculty of Medicine, Chiang Mai University Hospital, Chiang Mai, Thailand.

\section{Disclosure}

The authors report no conflict of interest in this work.

\section{References}

1. Besse B, Olaussen KA, Soria JC. ERCC1 and RRM1: ready for prime time? J Clin Oncol. 2013;31(8):1050-1060.

2. Fisher LA, Samson L, Bessho T. Removal of reactive oxygen speciesinduced 3'-blocked ends by XPF-ERCC1. Chem Res Toxicol. 2011; 24(11):1876-1881.

3. McVey M, Lee SE. MMEJ repair of double-strand breaks (director's cut): deleted sequences and alternative endings. Trends Genet. 2008; 24(11):529-538

4. Wang X, Zhao J, Yang L, et al. Positive expression of ERCC1 predicts a poorer platinum-based treatment outcome in Chinese patients with advanced non-small-cell lung cancer. Med Oncol. 2010;27(2):484- 490.

5. Ceppi P, Volante M, Novello S, et al. ERCC1 and RRM1 gene expressions but not EGFR are predictive of shorter survival in advanced non-small-cell lung cancer treated with cisplatin and gemcitabine. Ann Oncol. 2006;17(12):1818-1825.

6. Lord RV, Brabender J, Gandara D, et al. Low ERCC1 expression correlates with prolonged survival after cisplatin plus gemcitabine chemotherapy in non-small cell lung cancer. Clin Cancer Res. 2002;8(7): 2286-2291.

7. Pitterle DM, Kim YC, Jolicoeur EM, Cao Y, O’Briant KC, Bepler G. Lung cancer and the human gene for ribonucleotide reductase subunit M1 (RRM1). Mamm Genome. 1999;10(9):916-922.

8. Jordheim LP, Sève P, Trédan O, Dumontet C. The ribonucleotide reductase large subunit (RRM1) as a predictive factor in patients with cancer. Lancet Oncol. 2011;12(7):693-702.

9. Bepler G, Kusmartseva I, Sharma S, et al. RRM1 modulated in vitro and in vivo efficacy of gemcitabine and platinum in non-small-cell lung cancer. J Clin Oncol. 2006;24(29):4731-4737.

10. Reynolds C, Obasaju C, Schell MJ, et al. Randomized phase III trial of gemcitabine-based chemotherapy with in situ RRM1 and ERCC1 protein levels for response prediction in non-small-cell lung cancer. J Clin Oncol. 2009;27(34):5808-5815.

11. Goldstraw P, editor. IASLC Staging Manual in Thoracic Oncology. Orange Park, FL: Editorial Rx Press John Wiley \& Sons Ltd; 2009.

12. Goldstraw P. The 7th Edition of TNM in Lung Cancer: what now? J Thorac Oncol. 2009;4(6):671-673.

13. Travis WD, Brambilla E, Müller-Hermelink HK, Harris CC, editors. World Health Organization Classification of Tumors. Pathology and Genetics of Tumours of the Lung, Pleura, Thymus and Heart. Lyon, France: IARC Press; 2004.

14. Travis WD, Brambilla E, Noguchi M, et al. International association for the study of lung cancer/american thoracic society/european respiratory society international multidisciplinary classification of lung adenocarcinoma. J Thorac Oncol. 2011;6(2):244-285.

15. Tantraworasin A, Saeteng S, Lertprasertsuke N, Arreyakajohn N, Kasemsarn C, Patumanond J. Prognostic factors of tumor recurrence in completely resected non-small cell lung cancer. Cancer Manag Res. 2013;5:77-84.
16. Vilmar AC, Santoni-Rugiu E, Sørensen JB. ERCC1 and histopathology in advanced NSCLC patients randomized in a large multicenter phase III trial. Ann Oncol. 2010;21(9):1817-1824.

17. Tepeli E, Caner V, Büyükpınarbaşılı N, et al. Expression of ERCC1 and its clinicopathological correlations in non-small cell lung cancer. Mol Biol Rep. 2012;39(1):335-341.

18. Pesta M, Kulda V, Fiala O, et al. Prognostic significance of ERCC1, RRM1 and BRCA1 in surgically-treated patients with non-small cell lung cancer. Anticancer Res. 2012;32(11):5003-5010.

19. Olaussen KA, Dunant A, Fouret P, et al; IALT Bio Investigators. DNA repair by ERCC1 in non-small-cell lung cancer and cisplatin-based adjuvant chemotherapy. $N$ Engl J Med. 2006;355(10):983-991.

20. Lee KH, Min HS, Han SW, et al. ERCC1 expression by immunohistochemistry and EGFR mutations in resected non-small cell lung cancer. Lung Cancer. 2008;60(3):401-407.

21. Bepler G, Olaussen KA, Vataire AL, et al. ERCC1 and RRM1 in the international adjuvant lung trial by automated quantitative in situ analysis. Am J Pathol. 2011;178(1):69-78.

22. Custodio AB, González-Larriba JL, Bobokova J, et al. Prognostic and predictive markers of benefit from adjuvant chemotherapy in early-stage non-small cell lung cancer. J Thorac Oncol. 2009;4(7):891-910.

23. Booton R, Ward T, Ashcroft L, Morris J, Heighway J, Thatcher N. ERCC1 mRNA expression is not associated with response and survival after platinum-based chemotherapy regimens in advanced non-small cell lung cancer. J Thorac Oncol. 2007;2(10):902-906.

24. Roth JA, Carlson JJ. Prognostic role of ERCC1 in advanced non-smallcell lung cancer: a systematic review and meta-analysis. Clin Lung Cancer. 2011;12(6):393-401.

25. Friboulet L, Olaussen KA, Pignon JP, et al. ERCC1 isoform expression and DNA repair in non-small-cell lung cancer. $N$ Engl $\mathrm{J} \mathrm{Med}$. 2013;368(12):1101-1110.

26. Winton T, Livingston R, Johnson D, et al. National Cancer Institute of Canada Clinical Trials Group: National Cancer Institute of the United States Intergroup JBR 10 Trial Investigators. Vinorelbine plus cisplatin vs observation in resected non-small-cell lung cancer. $N$ Engl J Med. 2005;352(25):2589-2597.

27. Pignon JP, Tribodet H, Scagliotti GV, et al. Lung adjuvant cisplatin evaluation: a pooled analysis by the LACE Collaborative Group. J Clin Oncol. Jul 20, 2008;26(21):3552-3559.

28. Bepler G, Gautam A, McIntyre LM, et al. Prognostic significance of molecular genetic aberrations on chromosome segment $11 \mathrm{p} 15.5$ in non-small-cell lung cancer. J Clin Oncol. 2002;20(5):1353-1360.

29. Gautam A, Li ZR, Bepler G. RRM1-induced metastasis suppression through PTEN-regulated pathways. Oncogene. 2003;22(14): 2135-2142.

30. Bepler G, Sharma S, Cantor A, et al. RRM1 and PTEN as prognostic parameters for overall and disease-free survival in patients with nonsmall-cell lung cancer. J Clin Oncol. 2004;22(10):1878-1885.

31. Bepler G, Williams C, Schell MJ, et al. Randomized international phase III trial of ERCC1 and RRM1 expression-based chemotherapy versus gemcitabine/carboplatin in advanced non-small-cell lung cancer. J Clin Oncol. 2013;31(19):2404-2412.

32. Gong W, Zhang X, Wu J, et al. RRM1 expression and clinical outcome of gemcitabine-containing chemotherapy for advanced non-small-cell lung cancer: a meta-analysis. Lung Cancer. 2012;75(3):374-380.

33. Zheng Z, Chen T, Li X, Haura E, Sharma A, Bepler G. DNA synthesis and repair genes RRM1 and ERCC1 in lung cancer. $N$ Engl J Med. 2007;356(8):800-808 


\section{Publish your work in this journal}

Cancer Management and Research is an international, peer-reviewed open access journal focusing on cancer research and the optimal use of preventative and integrated treatment interventions to achieve improved outcomes, enhanced survival and quality of life for the cancer patient. The journal welcomes original research, clinical \& epidemiological

studies, reviews \& evaluations, guidelines, expert opinion \& commentary, case reports \& extended reports. The manuscript management system is completely online and includes a very quick and fair peerreview system, which is all easy to use. Visit http://www.dovepress.com/ testimonials.php to read real quotes from published authors.

Submit your manuscript here: http://www.dovepress.com/cancer-management-and-research-journal 